\title{
Microscale adaptive optics: wave-front control with a $\mu$-mirror array and a VLSI stochastic gradient descent controller
}

\author{
Thomas Weyrauch, Mikhail A. Vorontsov, Thomas G. Bifano, Jay A. Hammer, Marc Cohen, \\ and Gert Cauwenberghs
}

\begin{abstract}
The performance of adaptive systems that consist of microscale on-chip elements [microelectromechanical mirror ( $\mu$-mirror) arrays and a VLSI stochastic gradient descent microelectronic control system] is analyzed. The $\mu$-mirror arrays with $5 \times 5$ and $6 \times 6$ actuators were driven with a control system composed of two mixed-mode VLSI chips implementing model-free beam-quality metric optimization by the stochastic parallel perturbative gradient descent technique. The adaptation rate achieved was near 6000 iterations/s. A secondary (learning) feedback loop was used to control system parameters during the adaptation process, further increasing the adaptation rate. (C) 2001 Optical Society of America

OCIS codes: $\quad 010.0010,010.1080,230.3990$.
\end{abstract}

\section{Introduction}

Microelectromechanical system (MEMS) technology is a promising solution for resolving several obstacles that adaptive optics has faced during the past decade: system complexity, high cost, and difficulties in extending the spatial resolution of wave-frontaberration correction. From a MEMS point of view, adaptive optics is an important and challenging application that takes full advantage of the unique features of micromachined technology such as the ability to fabricate thousands of microscale mechanical actuators and optical elements (including lenses, lasers, and sensors) on a single silicon chip and the potential integration of this micromachined optical bench with control circuits and imaging sensors. ${ }^{1-3}$

Combined efforts in both adaptive optics and MEMS technologies can lead to the development of

T. Weyrauch and M. A. Vorontsov (mvorontsov@arl.army.mil) are with the U.S. Army Research Laboratory, Computational and Information Sciences Directorate, Adelphi, Maryland 20783. T. G. Bifano is with the Department of Manufacturing Engineering, Boston University, Brookline, Massachusetts 02446. J. A. Hammer is with MEMS Optical, Incorporated, 205 Import Circle, Huntsville, Alabama 35806. M. Cohen and G. Cauwenberghs are with the Department of Electrical and Computer Engineering, Johns Hopkins University, Baltimore, Maryland 21218.

Received 6 October 2000; revised manuscript received 28 April 2001.

0003-6935/01/244243-11\$15.00/0

(C) 2001 Optical Society of America affordable, high-resolution, fast microscale integrated adaptive-optics systems. Despite clearly outlined goals, the transition to MEMS-based adaptive optics is not a simple matter of replacing a conventional deformable mirror with an advanced micromachined mirror [or microelectromechanical mirror $(\mu$ mirror)].

A number of groups of researchers are now involved in developing micromachined adaptive mirrors. ${ }^{4-10}$ Unfortunately, newly developed $\mu$ mirrors are not often available for examination in actual adaptive-optics systems, and this makes comparing the performance of these devices difficult. Also, we have learned that MEMS-based adaptive optics is rather expensive. In most cases micromachined mirrors are unique, and only the possibility of mass production promises to bring down the cost of such mirrors; no one expects that $\mu$ mirrors will soon be as popular and in such demand as car airbag MEMS sensors.

There are also more fundamental problems related to the integration of MEMS and adaptive-optics technologies. Assume that high-resolution, good optical quality, inexpensive micromachined mirrors are available. Is conventional adaptive optics ready to accept these $\mu$-mirror innovations, leading to an entire adaptive system with high resolution, low cost, and small size? With traditional adaptive-optics approaches the transition to MEMS-based highresolution wave-front control will require the development of small, high-resolution wave-front 

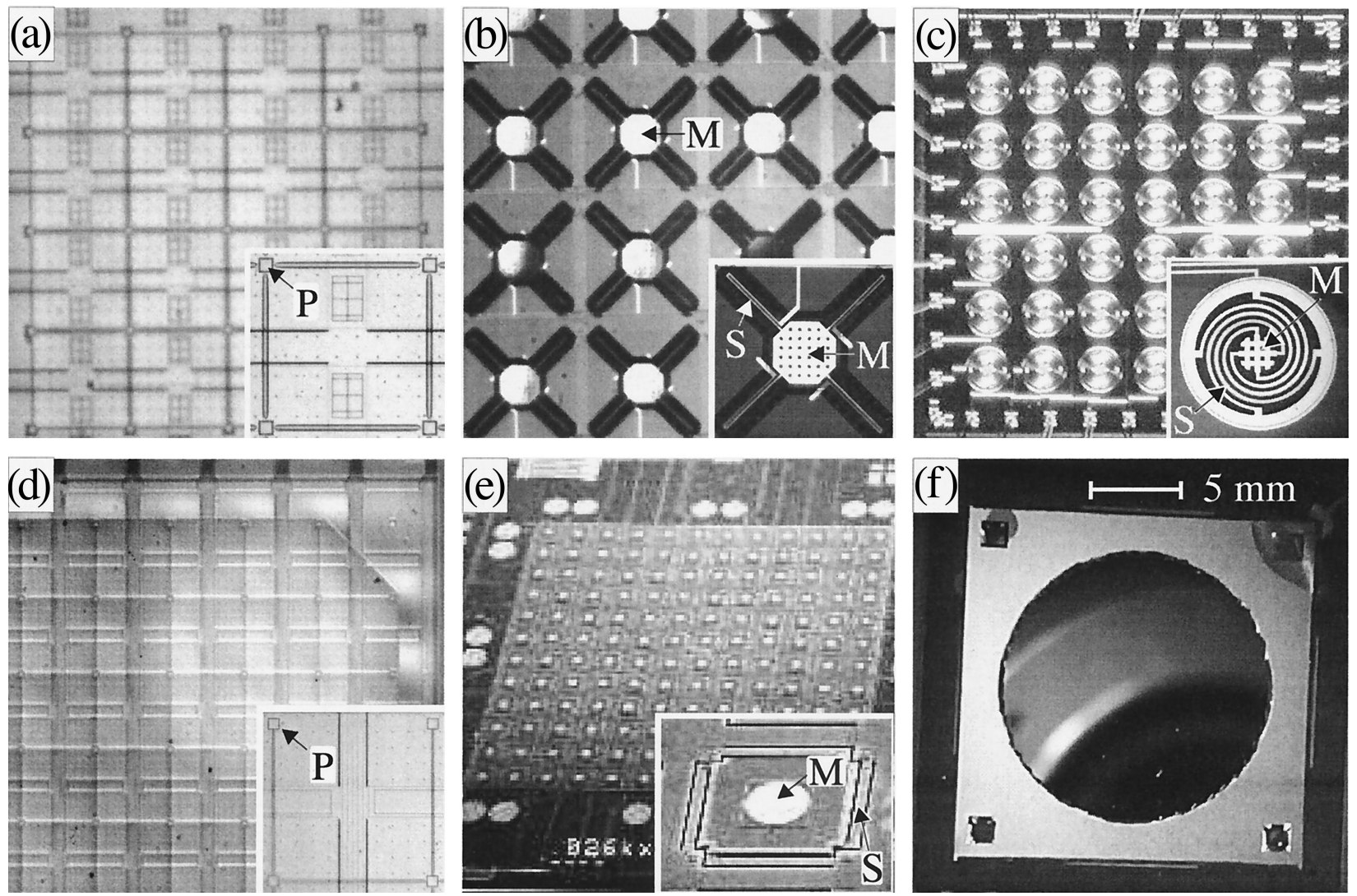

Fig. 1. Microphotographs of micromachined mirror arrays used in the experiments: (a) $\mathrm{BU}_{\mathrm{tt}}$ segmented membrane tip-tilt mirror with $4 \times 4$ elements $\left(5 \times 5\right.$ actuators), ${ }^{5}$ (b) $\mathrm{MO}_{z}$ piston-type mirror array with zig-zag spring, (c) $\mathrm{MO}_{s}$ piston-type mirror array with spiral spring, ${ }^{6}$ (d) $\mathrm{BU}_{12} 12 \times 12$ actuator tip-tilt mirror array, (e) UC piston-type $12 \times 12$ mirror array, ${ }^{7,17}$ (f) OKO continuous-membrane mirror. ${ }^{8}$ MO and UC mirrors are shown without lenslet arrays. Photographs of magnified mirror elements are shown at the bottom right in (a)-(e). Mirror array structural elements are marked by capital letters: $\mathrm{M}$, mirror elements; $\mathrm{S}$, spring (flexure); and $\mathrm{P}$, actuator post. Both BU $\mathrm{tt}_{\mathrm{t}}$ and $\mathrm{BU}_{12}$ are made without a metallic reflecting coating and thus the polysilicon membrane surface is partially transparent.

sensors and corresponding microscale phasereconstruction computational hardware. This cannot be easily achieved with existing wave-front sensing techniques. Thus the arrival of highresolution $\mu$-mirror arrays demands the development of new MEMS-friendly adaptive wave-front control techniques.

Among recent adaptive wave-front control algorithms the stochastic parallel gradient descent optimization technique is perhaps the most promising for MEMS-based adaptive optics. ${ }^{11-14}$ This algorithm does not require wave-front sensing and provides compact, low-power, scalable to high-resolution hardware implementation in a VLSI adaptive controller interfaced (or potentially integrated) with micromachined mirror arrays. Such a VLSI adaptive controller (e.g., the AdOpt control system ${ }^{15}$ ) has been developed and used in recent experiments with a 127-element liquid-crystal phase modulator and a 37control-channel continuously deformable micromachined mirror. ${ }^{16}$

The AdOpt control system architecture is ideal for evaluation of MEMS-based adaptive optics. Because stochastic parallel gradient descent control is model free and independent of $\mu$-mirror characteristics, different types of micromachined mirror device can be examined by use of the same adaptive system configuration. The high operational rate of the VLSI controller (up to $200 \mathrm{kHz}$ ) well exceeds the dynamic range of all existing $\mu$ mirrors, which makes the entire adaptation rate dependent only on the dynamic properties of the micromachined mirrors.

We begin this paper with an attempt to proceed with MEMS-based adaptive optics through the incorporation of recently developed micromachined mirror arrays and VLSI microelectronic control systems. Here we analyze and compare the performance of what are to our knowledge the first adaptive systems composed only of microscale on-chip elements: $\mu$-mirror arrays and a VLSI stochastic gradient descent microelectronic control system.

\section{Microelectromechanical Mirror Arrays}

Photographs of the micromachined mirror arrays used in the experiments described below are shown in Figs. 1(a)-1(e). For adequate comparison of adaptive-system performance we carried out the closed-loop experiments with $\mu$-mirror arrays that had approximately the same numbers of elements. These mirrors are the $5 \times 5$ element tip-tilt control $\mu$ mirror developed at Boston University (the $\mathrm{BU}_{\mathrm{tt}}$ mirror) in Fig. 1(a) and two types of $6 \times 6$ element 
piston-only control mirror made by MEMS Optical, Inc. (the $\mathrm{MO}_{z}$ and $\mathrm{MO}_{s}$ mirrors) shown in Figs. 1(b) and 1(c). The $\mathrm{BU}_{\mathrm{tt}}$ mirror in Fig. 1(a) is composed of a segmented silicone membrane supported by an underlying array of electrostatic parallel-plate actuators (posts) located at the mirror element corners. The mirror surface contains the print-through pattern that is visible in Fig. 1(a). The $\mathrm{MO}_{z}$ and $\mathrm{MO}_{s}$ mirrors in Figs. 1(b) and 1(c) have differently shaped springs $S$ holding mirror elements $M$ : zigzag for the $\mathrm{MO}_{z}$ and spiral-shaped for the $\mathrm{MO}_{s}$ mirrors.

We also examined (in an open-loop system only) the characteristics of two $12 \times 12$ mirror arrays developed at Boston University [tip-tilt-type $\mathrm{BU}_{12}$ mirror in Fig. 1(d)] and of one from the University of Colorado at Boulder [piston-type UC mirror shown in Fig. 1(e)] and a 37-electrode micromachined deformable mirror from OKO Technologies [Fig. 1(f)]. To increase the fill factor and partially overcome problems related to the optical quality of the mirror surfaces we used all mirror arrays except the BU $\mu$ mirrors and the OKO mirror with a lenslet array attached to the $\mu$-mirror chip. The experiments showed that a lenslet array composed of short (6-mm or less) focallength lenses introduces additional phase distortions (defocus and spherical aberrations) that cannot be compensated for by the adaptive system itself. In addition, use of the lenslet array typically provides a fill factor of less than $75 \%$ and requires rather precise adjustment, to ensure $90^{\circ}$ illumination of the mirror surface.

The interference and focal-plane intensity patterns presented in Fig. 2 illustrate the optical quality of the micromachined mirrors in the absence of applied voltages. The elements of the $\mathrm{BU}_{\mathrm{tt}}$ mirror array in Figs. 2(a) and 2(b) display the presence of a rather strong unwanted curvature. In the more-recent $\mu$ mirror $\left[\mathrm{BU}_{12}\right.$ mirror in Figs. 2(c) and 2(d)] this curvature was almost completely eliminated by ioninduced compression of the mirror surface. The typical interference and focal-plane intensity patterns of the $\mathrm{MO}_{s}$ mirror with the lenslet array in Figs. 2(e) and 2(f) display the presence of severe wavefront aberrations that result from the optical quality of both the mirror surface and the lenslet array. The $\mathrm{MO}_{z}$ mirror with the lenslet array had an optical quality similar to that shown in Figs. 2(e) and 2(f).

Typically, $\mu$-mirror electromechanical characteristics are described in terms of voltage-deflection curves (the dependence of the actuator's deflection on applied voltage).5,18 For analysis of the adaptive system based on performance metric optimization described below, it is more appropriate to use a different characteristic, which we call Strehl ratio sensitivity curves. We estimated the electromechanical characteristics of the $\mu$-mirror elements by measuring the dependence of Strehl ratio (St) on voltages $u_{j}(j=$ $1, \ldots, N)$ applied to various $\mu$-mirror electrodes $(N$ is the number of actuators). The Strehl ratio is defined as $\operatorname{St}\left(u_{j}\right)=P\left(u_{j}\right) / P_{0}$, where $P\left(u_{j}\right)$ and $P_{0}=$ $P\left(u_{j}=0\right)$ are optical power values measured inside a $50-\mu \mathrm{m}$ pinhole placed in the focal plane of a lens with
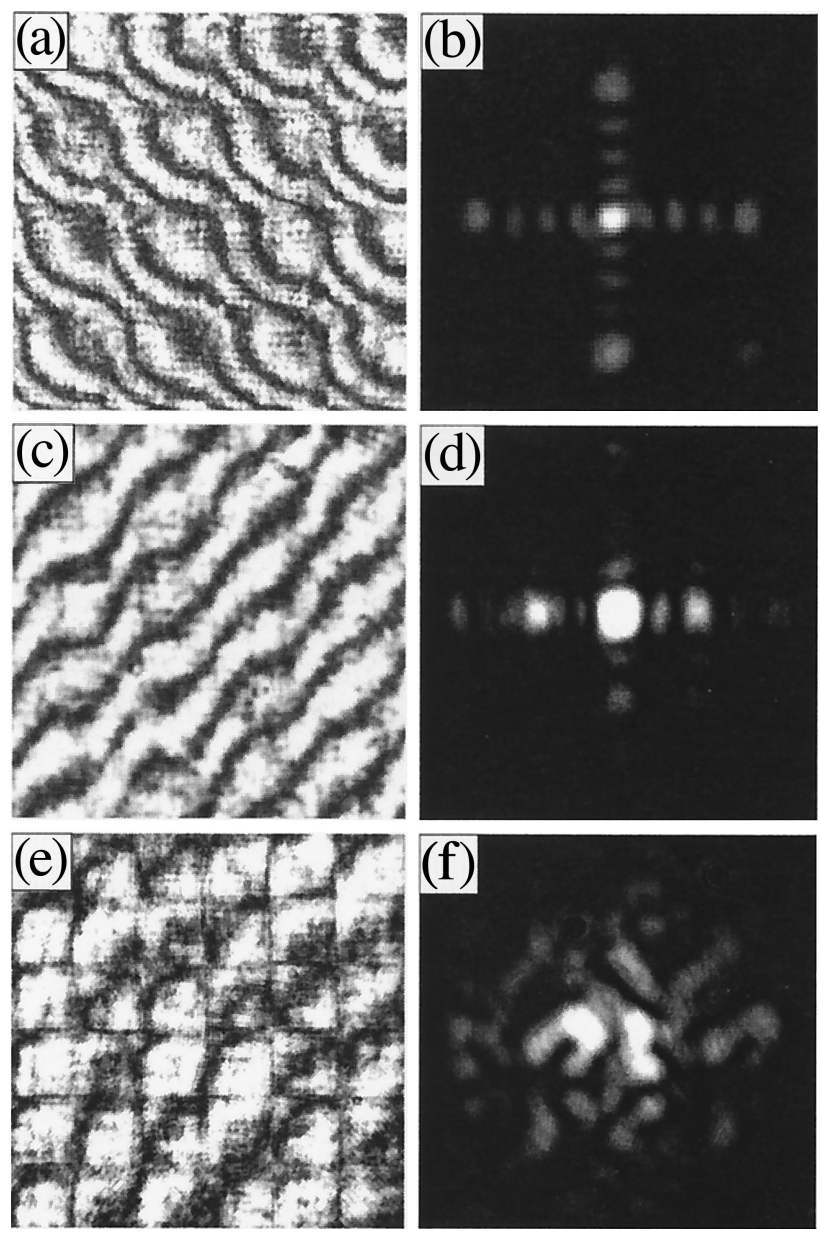

Fig. 2. (a), (c), (e) Mirror surface interference patterns and (b), (d), (f) corresponding far-field intensity distributions with no applied voltages for (a), (b) the $\mathrm{BU}_{\mathrm{tt}}$ mirror, (c), (d) the $\mathrm{BU}_{12}$ mirror, and (e), (f) the $\mathrm{MO}_{s}$ mirror with a diffractive lenslet array $(\mathrm{e}, \mathrm{f})$.

focal length $F=15 \mathrm{~cm}$ (Fig. 3). Results of the Strehl ratio measurements are presented in Fig. 4 for $\mathrm{MO}_{s}$ and for $\mathrm{BU}_{\mathrm{tt}}$ mirror chips for three different actuator locations. For the piston-type $\mathrm{MO}_{s}$ mirror the dependencies $\operatorname{St}\left(u_{j}\right)$ are periodic functions [Fig. 4(a)] with approximately equal-amplitude maxima that correspond to $2 \pi$ rad phase shifts. As can be seen from Fig. 4(a), the mirror stroke depends on the actuator's location and varies from $\sim 1.27 \mu \mathrm{m}(3.75 \pi$ phase shift) for the corner element to $1.5 \mu \mathrm{m}$ for the actuator located in the mirror center. The Strehl ratio sensitivity curves for the tip-tilt mirror array $\left(\mathrm{BU}_{\mathrm{tt}}\right.$ mirror) presented in Fig. 4(b) are quite different

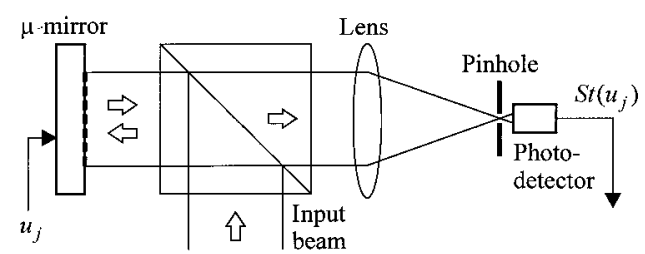

Fig. 3. Schematic of the experimental setup for Strehl ratio measurements. 

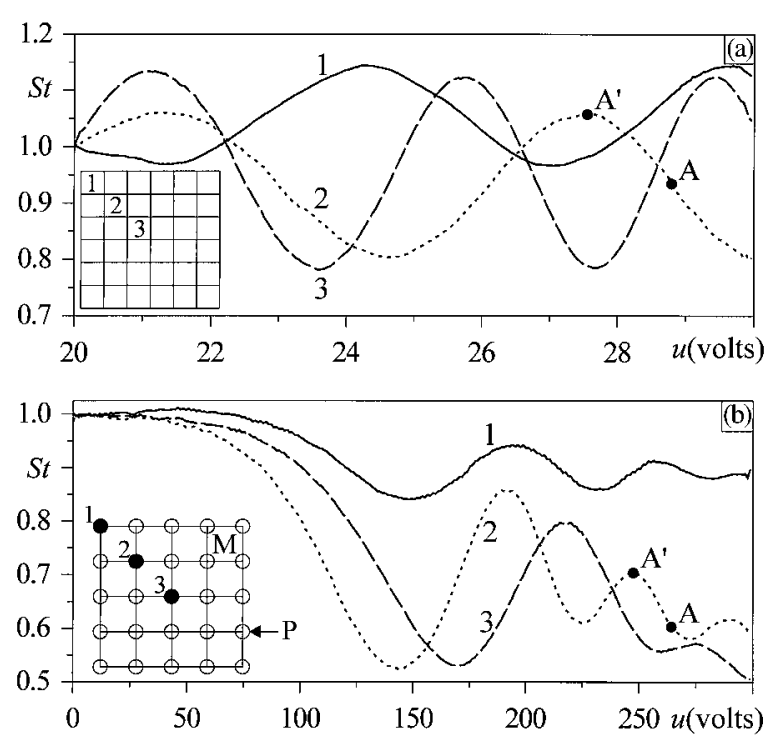

Fig. 4. Dependence of Strehl ratio on voltage applied to a single actuator for (a) the $\mathrm{MO}_{s}$ and (b) the $\mathrm{BU}_{\mathrm{tt}}$ mirrors. Strehl ratio sensitivity curves 1,2 , and 3 correspond to three actuator locations within the mirror array, as shown at the bottom left ( $M$ is a mirror element; $\mathrm{P}$ is an actuator post).

from the corresponding curves for the piston-type mirror [cf. the curves in Figs. 4(a) and 4(b)]. These curves are also periodic with $2 \pi$ rad phase shifts between local maxima, but, Fig. 4(b) shows, the amplitude of the Strehl ratio local maximum decreases with an increase in the actuator displacement. The dependence of the sensitivity curve on actuator location is more pronounced for the tip-tilt mirror than for the piston-type mirror in Fig. 4(a). Spatial nonuniformity in the actuator sensitivity and the presence of local maxima complicates control-system design, especially if the corresponding $\mu$ mirror is used in a phase-conjugation-type adaptive optical system. ${ }^{19}$

A technique similar to the one described above was used for evaluation of the $\mu$-mirror elements' dynamic characteristics. In the scheme in Fig. 3 a small-amplitude probe voltage $u(t)=a \sin (v t)+a_{0}$ was applied to a single $\mu$-mirror actuator, where $a$ and $v$ are the ac component amplitude and frequency, respectively, and $a_{0}$ is an offset voltage. The measured dependencies of Strehl ratio amplitude $\mathrm{St}(v)$ on frequency $v$ (Strehl ratio resonance curves) are shown in Fig. 5 for several $\mu$-mirror types. The first resonance was observed at frequencies $v_{0} \cong 2 \mathrm{kHz}$ for the OKO mirror, $v_{0} \cong 5.3 \mathrm{kHz}$ for the $\mathrm{MO}_{s}$ mirror, and $v_{0}$ $\cong 5.8 \mathrm{kHz}$ for the $\mathrm{MO}_{z}$ mirror. For both the $\mathrm{BU}_{\mathrm{tt}}$ and the UC mirrors the observed dependencies $\operatorname{St}(v)$ had no resonance peaks within the examined frequency bands of 100 and $20 \mathrm{kHz}$, respectively. The $\mathrm{BU}_{\mathrm{tt}}$ mirror chip displayed relatively uniform dynamic characteristics within a frequency bandwidth of $\sim 10 \mathrm{kHz}$. The major characteristics of the $\mu$ mirrors that we examined are summarized in Table 1.

Based on the previous analysis, the following $\mu$ mirrors were chosen for closed-loop experiments with the microscale (MEMS-VLSI) adaptive system: the

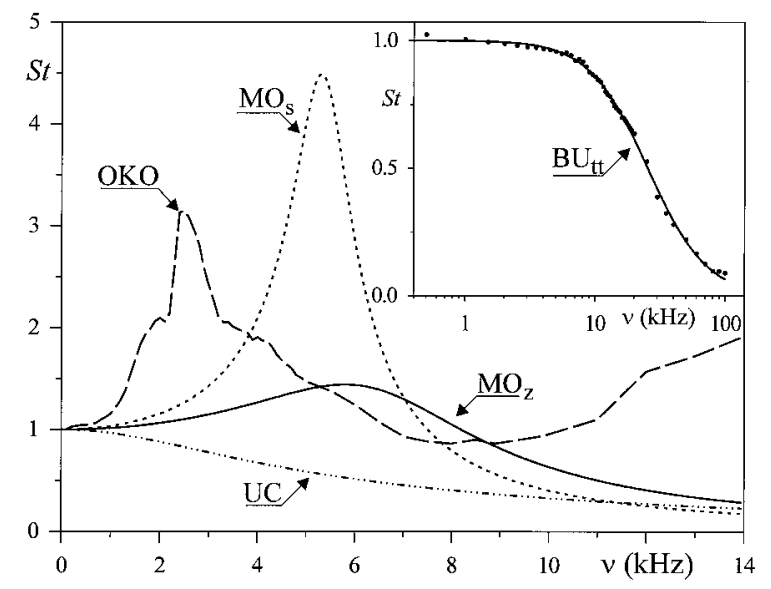

Fig. 5. Strehl ratio resonance curves for the micromachined mirrors examined. Resonance curves for $\mathrm{OKO}, \mathrm{UC}, \mathrm{MO}_{s}$, and $\mathrm{MO}_{z}$ mirrors are shown in linear scales and the BU mirror on a logarithmic frequency scale.

tip-tilt type $5 \times 5$ mirror array from Boston University (BU $\mathrm{BU}_{\mathrm{tt}}$ mirror) and two piston-type $6 \times 6$ mirror arrays from MEMS Optical, Inc. (the $\mathrm{MO}_{s}$ and $\mathrm{MO}_{z}$ mirrors). The rationale behind this choice is as follows: The $\mathrm{BU}_{\mathrm{tt}}$ mirror chip provides the best dynamic operational range among the $\mu$ mirrors examined, but it cannot be interfaced directly with the AdOpt VLSI controller in its present form, fabricated in a standard complementary metal-oxide semiconductor process. The output control voltages from the VLSI controller chips are within the range of -5 to $+5 \mathrm{~V}$. These voltages are not sufficiently high to drive the $\mathrm{BU}_{\mathrm{tt}}$ mirror array. As can be seen from the sensitivity curves in Fig. 4(b), the $\mathrm{BU}_{\mathrm{tt}}$ mirror requires an $\sim 200-\mathrm{V}$ control voltage range to provide a $2 \pi$ phase shift. For this reason amplifiers with output voltages in the range $0-300 \mathrm{~V}$ were used to interface the $\mathrm{BU}_{\mathrm{tt}}$ mirror with the VLSI controller. The set of 26 amplifiers developed at Boston University was built onto one $6.5^{\prime \prime} \times 4.5^{\prime \prime}(16.51 \mathrm{~cm} \times 11.43$ $\mathrm{cm})$ board. The need for external high-voltage amplifiers is highly undesirable for the future development of high-resolution microscale adaptive systems. From this point of view the low-voltage MO and UC mirrors have the obvious advantage (unless highvoltage amplifiers are integrated onto the MEMS chip): both of these mirrors can be interfaced with the VLSI controller directly to form a microscale adaptive system.

In the closed-loop experiments with MO mirrors an additional constant (offset) voltage $u_{0}$ was applied to the mirrors' common electrode to permit direct interfacing of the VLSI controller with the $\mathrm{MO}_{s}$ mirror array. With the offset voltage $u_{0}=-25 \mathrm{~V}$, the $\mathrm{MO}_{s}$ mirror operated with input control voltages that ranged from 20 to $30 \mathrm{~V}$ and provided an approximately $4 \pi$ wave-front phase shift [see the sensitivity curves in Fig. 4(a)]. For the $\mathrm{MO}_{z}$ mirror an offset voltage of $-10 \mathrm{~V}$ was sufficient to provide a phase shift near $3.5 \pi$, with the control voltage ranging from 5 to $15 \mathrm{~V}$. 
Table 1. Parameters of the $\mu$-Mirror Chips Used in the Experiments

\begin{tabular}{|c|c|c|c|c|c|c|c|}
\hline Mirror & Type of Motion & $\begin{array}{l}\text { Number of } \\
\text { Actuators }\end{array}$ & $\begin{array}{c}\text { Mirror } \\
\text { Element Size }\end{array}$ & Actuator Pitch & $\begin{array}{c}\text { Actuator } \\
\text { Voltage } \\
\text { Range (V) }\end{array}$ & $\begin{array}{l}\text { Stroke } \\
(\mu \mathrm{m})\end{array}$ & $\begin{array}{l}\text { Resonance } \\
\text { Frequency }\end{array}$ \\
\hline $\mathrm{MO}_{z}$ & Piston & 36 & $160 \mu \mathrm{m}$ & $500 \mu \mathrm{m}$ (rectangular) & $0-15$ & 0.7 & $5.8 \mathrm{kHz}$ \\
\hline $\mathrm{MO}_{s}$ & Piston & 36 & $100 \mu \mathrm{m}$ & $500 \mu \mathrm{m}$ (rectangular) & $0-30$ & 1.1 & $5.3 \mathrm{kHz}$ \\
\hline $\mathrm{UC}$ & Piston & 128 (36 used) & $74 \mu \mathrm{m}$ & $250 \mu \mathrm{m}$ (rectangular) & $0-11$ & 0.9 & Not observed \\
\hline $\mathrm{BU}_{\mathrm{tt}}$ & Tip-tilt & 25 & $242 \mu \mathrm{m}$ & $250 \mu \mathrm{m}$ (rectangular) & $0-300$ & 0.9 & Not observed \\
\hline $\mathrm{OKO}$ & $\begin{array}{l}\text { Continuous } \\
\text { membrane }\end{array}$ & 37 & $\begin{array}{l}\text { 12-mm active } \\
\text { aperture }\end{array}$ & $1.75 \mathrm{~mm}$ (hexagonal) & $0-210$ & 6 (center) & $2 \mathrm{kHz}$ (1st peak) \\
\hline
\end{tabular}

\section{Microscale MEMS-VLSI Adaptive System}

\section{A. Experimental Setup}

A schematic of the microscale VLSI-MEMS-based adaptive system is shown in Fig. 6. Key system elements include a micromachined mirror, a VLSI controller, and a photodetector, as presented in Fig. 7. The expanded input laser beam from the semiconductor laser $(\lambda=0.69 \mu \mathrm{m})$ with a diameter of $\sim 8 \mathrm{~mm}$ was split into two equal parts by beam splitter $\mathrm{BS}_{1}$ (Fig. 6). Reference mirror $M$ and micromachined mirror $\mu-M$ formed an interferometer that was used to visualize the wave-front phase pattern in the plane of camera $\mathrm{CCD}_{1}$. An imaging system composed of lenses $L_{1}$ and $L_{2}$ formed a magnified image of the $\mu$-mirror surface at the camera chip. The wave reflected from the $\mu$ mirror was focused by lens $L_{1}$ and beam splitter $\mathrm{BS}_{2}$ onto the plane of the $50-\mu \mathrm{m}$ pinhole. To prevent the reference beam from entering the pinhole we slightly tilted the reference mirror. The laser beam's power inside the pinhole was measured with a photodetector, and the photodetector's output voltage was used as the adaptive system's performance metric (beam-quality metric) $J$. This beam-quality metric is proportional to the previously defined Strehl ratio St and depends on voltages $\left\{u_{j}\right\}$ applied to the $\mu$-mirror electrodes: $J=J\left(u_{1}, \ldots\right.$, $\left.u_{N}\right)$, where $N=25$ for $\mathrm{BU}_{\mathrm{tt}}$ and $N=36$ for $\mathrm{MO}$ mirrors. Accordingly, maximization of beamquality metric $J$ is equivalent to Strehl ratio maximization. Lens $\mathrm{L}_{3}$ and beam splitter $\mathrm{BS}_{3}$ were used

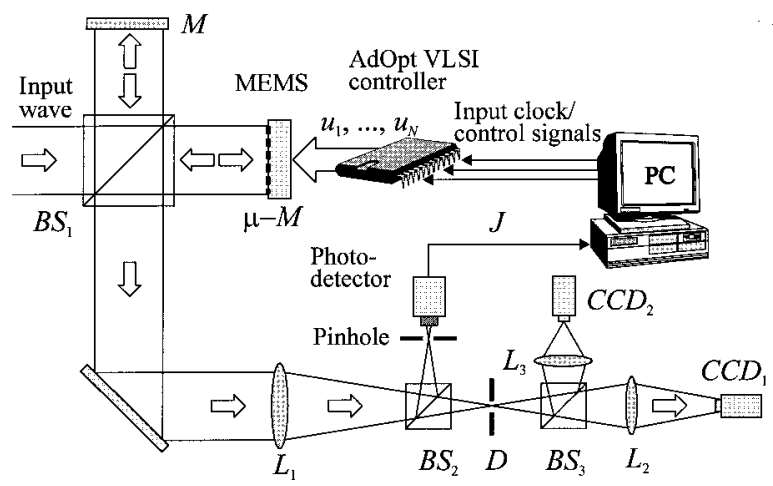

Fig. 6. Schematic of the microscale adaptive-optics system based on the AdOpt VLSI controller and micromachined mirrors used in the experiments. to magnify the image of the laser beam's intensity distribution in the plane conjugate to the pinhole. This intensity distribution was registered by camera $\mathrm{CCD}_{2}$. Iris diaphragm D placed in the focal plane of lens $\mathrm{L}_{1}$ was used as a low-pass spatial filter to cut off the higher-order spectral components that resulted from laser beam diffraction off the periodic structure of the $\mu$-mirror array.

\section{B. VLSI Feedback Controller}

Adaptive feedback control of the $\mu$-mirror arrays was achieved with VLSI implementation of the parallel stochastic perturbative gradient descent algorithm (AdOpt control system architecture). ${ }^{15,16}$ The VLSI controller performed iterative parallel computation (upgrade) of control voltages $\left\{u_{j}\right\}$ applied to the $\mu$-mirror electrodes, leading to maximizationminimization of the externally supplied performance metric $J$. A single AdOpt chip provided parallel updates for 19 output-control signals. Correspondingly, two AdOpt chips were enough to control the micromachined mirror arrays used in the experi-

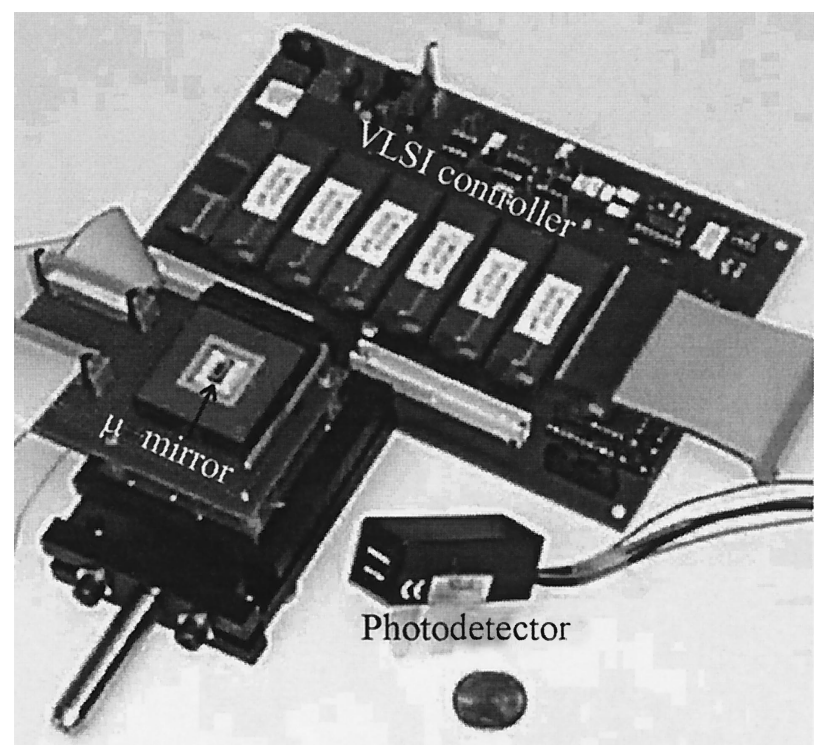

Fig. 7. Photograph of the key elements (VLSI controller board, $\mu$ mirror, and photodetector) of the microscale adaptive-optics system based on stochastic parallel gradient descent optimization. The VLSI controller board comprises 7 AdOpt chips and can provide control of as many as 133 channels. The U.S. quarter coin at the bottom right is used to indicate the scale. 


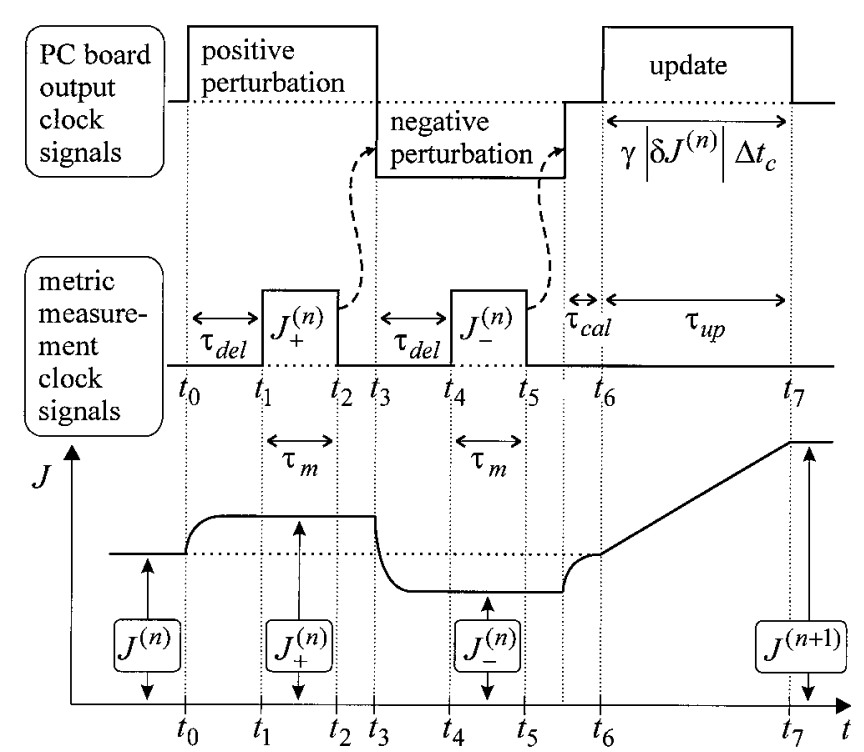

Fig. 8. Simplified timing diagrams for a single iteration cycle of the AdOpt system: sequence of external clock signals supplied to the AdOpt chips (top), clock signals used for beam-quality metric measurements (middle), corresponding sequence of changes in beam-quality metric $J$ (bottom).

ments. The VLSI controller requires both analog and digital externally supplied control signals generated by a desktop PC with two analog-digital inputoutput cards (ComputerBoards CIO-DAS1602/12). The computer generated the timing signals, controlled measurements of beam-quality metric $J$, and updated the algorithm parameters. Controlling the VLSI system parameters permitted implementation of a secondary control-loop system designed to modify the algorithm parameters during the adaptation process to increase its convergence rate (see Subsection 4.D below). Using a computer to control the AdOpt system was convenient for analysis of system performance. In actual microscale systems the computer can be replaced by a microcontroller integrated on the AdOpt board.

As an illustration of VLSI controller operational principles, consider the simplified timing diagram shown in Fig. 8. A single iteration cycle of the control voltage's update at the $n$th iterative step consists of the following phases:

(1) A clock signal from the computer board is applied to the VLSI controller digital input at the moment $t=$ $t_{0}$. This signal activates on-chip generation of an uncorrelated pseudorandom parallel stream of coin-flip signals (perturbations) $\left\{\delta u_{j}^{(n)}\right\}$ that have identical amplitudes $\left|\delta u_{i}^{(n)}\right|=\pi$ and a Bernoulli probability distribution $P\left[\delta u_{j}^{(n)}=+\pi\right]=0.5$ for all $j=1, \ldots N$ and all $n$. These perturbations are applied in parallel to the VLSI system's output electrodes interfaced directly with the micromachined mirror electrodes (for $\mathrm{MO}_{s}$ and $\mathrm{MO}_{z}$ mirrors) or through amplifiers (for the $\mathrm{BU}_{\mathrm{tt}}$ mirror). Voltage perturbations result in a variation in the laser beam power inside the pinhole and hence in a variation of the photodetector's output voltage that corresponds to beam-quality metric $J$. The perturbed beam-quality metric value $J_{+}^{(n)}=J\left[u_{1}^{(n)}+\right.$ $\left.\delta u_{1}^{(n)}, \ldots, u_{N}^{(n)}+\delta u_{N}^{(n)}\right]$ is measured at the moment $t_{1}=t_{0}+\tau_{\text {del }}$ after a time delay $\tau_{\text {del }}$ introduced by the PC computer. The controllable time delay was introduced to prevent $\mu$-mirror dynamics from influencing metric measurement: $\tau_{\text {del }} \approx 1.5 \tau_{\mu}$, where $\tau_{\mu}=2 \pi / \nu_{0}$ is the $\mu$ mirror's characteristic response time and $v_{0}$ is resonance frequency.

(2) When metric value $J_{+}^{(n)}$ has been measured (at $t_{2}=t_{1}+\tau_{m}$, where $\tau_{m} \cong 45 \mu \mathrm{s}$ ), the computer board activates on-chip generation of complementary perturbations $\left\{-\delta u_{j}^{(n)}\right\}$ at moment $t_{3}$. These perturbations applied to the $\mu$-mirror array result in a corresponding change in beam-quality metric $J_{-}^{(n)}=$ $J\left[u_{1}^{(n)}-\delta u_{1}^{(n)}, \ldots, u_{N}^{(n)}-\delta u_{N}^{(n)}\right]$.

(3) After the computer introduces time delay $\tau_{\text {del }}$, the perturbed beam-quality metric value $J_{-}^{(n)}$ is measured and digitized. In the timing diagram in Fig. 8 this measurement corresponds to moment $t_{4}=t_{3}+$ $\tau_{\text {del }}$. After the measurement (at time $t_{5}=t_{4}+\tau_{m}$ ), perturbation voltages are deactivated.

(4) Using perturbed beam-quality metric values $J_{+}^{(n)}$ and $J_{-}^{(n)}$, the computer calculates the following quantities: metric perturbation, $\delta J^{(n)}=J_{+}^{(n)}-J_{-}^{(n)}$; averaged metric, $J^{(n)}=1 / 2\left[J_{+}^{(n)}+J_{-}^{(n)}\right]$; $\operatorname{sign}\left[\delta J^{(n)}\right]$; and the product of $\gamma \delta J^{(n)}$, where $\gamma$ is a predefined upgrade coefficient. With a $400-\mathrm{MHz}$ PC the time required for these calculations, $\tau_{\text {cal }}$, is less than 0.1 $\mu \mathrm{s}$. Parameter $\gamma$ controls the update rate and the optimization mode of the performance metric: Positive $\gamma$ corresponds to metric maximization; negative, to metric minimization. The product $\gamma \delta \delta J^{(n)}$ is used to generate the update pulse $\tau_{\text {up }}=\gamma\left|\delta J^{(n)}\right| \Delta t_{c}$ applied to the VLSI chip input, where $\Delta t_{c}$ is the duration of the clock pulses. In the feedback control system described here, $\Delta t_{c} \cong 1.0 \mu \mathrm{s}$.

The control-voltage update phase starts at time $t_{6}$ and lasts a duration of $\tau_{u p}$ (typically $\tau_{u p}$ varies from $\Delta t_{c}$ to $25 \Delta t_{c}$ ). During this time the VLSI controller computes updated control-output voltages according to the following gradient descent procedure:

$$
u_{j}^{(n+1)}=u_{j}^{(n)}+\gamma^{\prime}\left|\delta J^{(n)}\right| \operatorname{sign}\left[\delta J^{(n)}\right] \operatorname{sign}\left[\delta u_{j}^{(n)}\right],
$$

where $\gamma^{\prime}$ is a constant that is proportional to $\gamma$. Using the AdOpt system, one can obtain the outputvoltage change for each control channel [the second term in Eq. (1)] by charging-discharging a set of on-chip capacitors during the update time $\tau_{\text {up }}=$ $\gamma\left|\delta J^{(n)}\right| \Delta t_{c}$. The value sign[ $\left[\delta J^{(n)}\right]$ is supplied to the chip input through the PC board, and the values $\operatorname{sign}\left[\delta u_{j}^{(n)}\right]$ are stored on-chip.

With the use of a $1.0-\mathrm{MHz}$ external-clock generator and two analog-digital input-output boards (ComputerBoards CIO-DAS1602/12), one iteration of the control-voltages update required $\tau_{\text {it }} \approx 160 \mu \mathrm{s}$ (in the absence of the computer-introduced time delay $\tau_{\text {del }}$ ). Using commercially available fast analog-digital converters, one can further decrease the time required for a single iteration to $\tau_{\text {it }} \approx 100 \mu \mathrm{s}$. 


\section{Self-Induced Phase-Distortion Compensation}

A. Characterization of Adaptive-System Performance

Adaptive-system performance was characterized by the self-induced aberration-compensation technique described in Refs. 15 and 16 . In this method random phase distortions are introduced by an adaptive system as a result of minimization of the beam-quality metric. For statistical analysis of system operation we used a large number $M$ of consecutively repeated adaptation trials (typically $M=500$ ). Each adaptation trial included a sequence of $N_{\text {it }}=4096$ iteration steps $n$ (control-voltage updates) executed by the AdOpt system $\left(n=1, \ldots, N_{\text {it }}\right)$. During the first 2048 iteration steps the update parameter $\gamma$ set by the computer was negative, which corresponded to minimization of the beam-quality metric. This adaptation mode was used to generate random phase distortions that resulted in a highly distorted laser beam intensity distribution in the plane of the photodetector. At the iteration $n=\left(1 / 2 N_{\text {it }}+1\right)=2049$ the sign of update parameter $\gamma$, and hence the adaptation mode, was changed. At this second phase of the adaptation trial (from $n=2049$ to $n=4096$ ) the adaptive system maximized the beam-quality metric such that the laser beam's power was concentrated inside the pinhole. At each iteration the measured perturbed beam-quality metric values $J_{+}^{(n)}$ and $J_{-}^{(n)}$ were averaged: $J^{(n)}=\left[J_{+}^{(n)}+J_{-}^{(n)}\right] / 2$. The metric values $\left\{J^{(n)}\right\}(n=1, \ldots, 4096)$ as well as the metric perturbation values $\left\{\delta J^{(n)}\right\}$ were stored in computer memory. The complete set of data used for statistical analysis of adaptive-system performance included metric values $\left\{J^{(n)}\right\}$ and $\left\{\delta J^{(n)}\right\}$ collected for $M$ consecutively repeated adaptation trials.

\section{B. Convergence Rate and Adaptation Speed}

An example of typical dependence of metric $J$ on iteration number $n$ (adaptation evolution curve) obtained during a single minimization-maximization trial is shown in Fig. 9(a) for the closed-loop system with the $\mathrm{BU}_{\mathrm{tt}}$ mirror. Note that the optimization mode (sign of the update coefficient $\gamma$ ) was changed from metric maximization to minimization at $n=1$ and vice versa at $n=2049$. The iteration number $n=$ 0 corresponds to the last iteration of the previous trial $(n=4096)$. The adaptation evolution curve $\langle J(n)\rangle$ averaged over all 500 trials is presented in Fig. 9(b). From the evolution curve $\langle J(n)\rangle$ one can estimate an adaptation convergence rate, defined here as the average number of iterations $n_{c}$ max (or $n_{c}{ }^{\min }$ ) required for achieving $80 \%$ of the maximum (minimum) level of the beam-quality metric. From the adaptation evolution curve in Fig. 9(b), the convergence rate is approximately $n_{c}$ max $\approx 60$ iterations for metric maximization and $n_{c}{ }^{\min } \approx 140$ iterations for metric minimization.

The difference in adaptation rates (beam-quality metric maximization occurs on average twice as fast as metric minimization) is due to two major factors. The first is the decrease in signal-to-noise ratio that occurs during metric minimization, which slows down the adaptation process. The second factor is
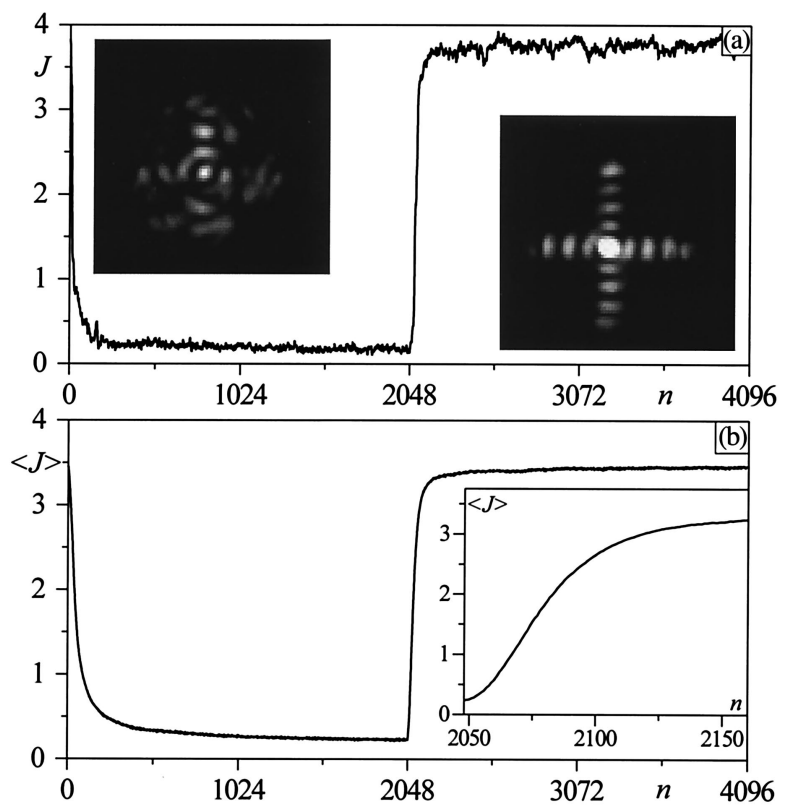

Fig. 9. Experimental results of self-induced phase-distortion compensation in an adaptive system with the $\mathrm{BU}_{\mathrm{tt}}$ mirror: adaptation evolution curves for optimization of the beam-quality metric (a) for a single trial and (b) after averaging over 500 trials. Photographs correspond to focal-plane intensity distributions at the end of minimization (left) and maximization (right). The transition process ( $\sim 60$ iteration long) is shown in (b) as an inset.

the existence of a large number of local minima such that the adaptive system may be trapped during the minimization process. This problem is discussed in Subsection 4.C below.

The characteristic adaptation time $\tau_{\text {ad }}$ is the product of convergence rate $n_{c}{ }^{\max }$ and time $\tau_{\text {it }}$ required for a single iteration of control-voltage update $\left(\tau_{\text {it }}\right.$ is dependent on time delay $\tau_{\text {del }}$ introduced by the computer): $\tau_{\text {ad }}=n_{c}{ }^{\max } \tau_{\text {it. }}$. In the experiments with different $\mu$ mirrors, we used the minimum possible time-delay value $\tau_{\text {del }}$ for each mirror that still ensured stable adaptation. This minimal time-delay value depended on the $\mu$ mirror's frequency bandwidth. For the fastest $\left(\mathrm{BU}_{\mathrm{tt}}\right)$ mirror this time delay was set to zero. The averaged adaptation evolution curves $\langle J(t)\rangle$ for the system with $\mathrm{BU}_{\mathrm{tt}}, \mathrm{MO}_{z}$, and $\mathrm{MO}_{s}$ are presented in Fig. 10 as functions of physical time $t$. The shortest adaptation time $\tau_{\text {ad }}(\approx 10 \mathrm{~ms})$ was achieved with the $\mathrm{BU}_{\mathrm{tt}}$ mirror array. The limiting factor for the $\mathrm{BU}_{\mathrm{tt}}$ mirror was not the mirror's mechanical bandwidth $(\approx 10 \mathrm{kHz})$ but the speed of the computer boards. For the $\mathrm{MO}_{z}$ and $\mathrm{MO}_{s}$ mirrors the adaptation time was limited by mechanical resonance of the mirror actuators, with $\tau_{\text {ad }} \approx 105-120 \mathrm{~ms}$ for both MO mirrors (see the resonance curves in Fig. 5). The photos in Fig. 10 show intensity distributions in the focal plane (plane of the pinhole in Fig. 6) obtained in the adaptive system with an $\mathrm{MO}_{s}$ mirror array. Metric minimization typically led to the appearance of a dark spot in the pinhole, as shown in Fig. 10 (left photo), whereas metric maximization always resulted in laser beam concentration in the pin- 


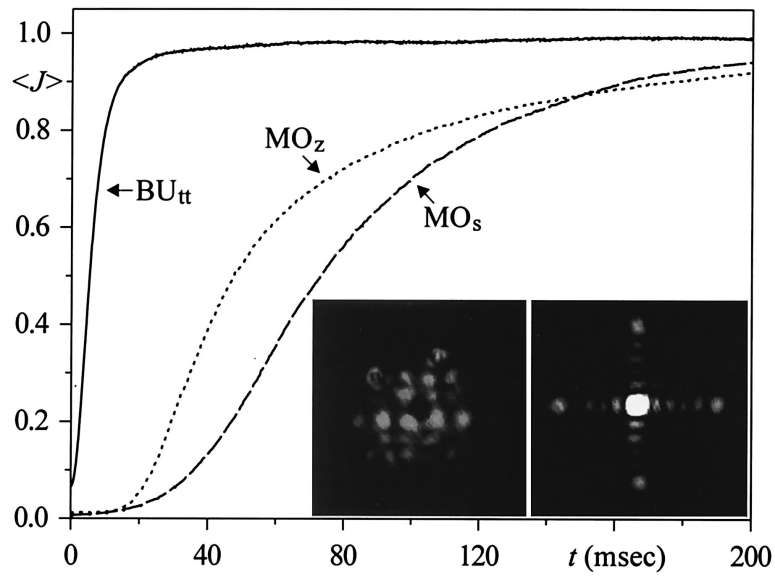

Fig. 10. Beam-quality metric evolution curves averaged over 500 maximization trials $\langle J(t)\rangle$ for the following $\mu$-mirror arrays: $\mathrm{BU}_{\mathrm{tt}}$, $\mathrm{MO}_{s}$, and $\mathrm{MO}_{z}$. Photographs show typical snapshots of focalplane intensity distributions for the $\mathrm{MO}_{s}$ mirror at (left) $t=0$ and (right) $t=200 \mathrm{~ms}$.

hole area [Fig. 10 (right photo)]. Note that the estimated adaptation time $\tau_{\text {ad }}$ represents the rather pessimistic situation when the adaptive system is compensating for specially prepared temporally uncorrelated random phase distortions that correspond to the most severe wave-front aberrations that can be created with the $\mu$ mirror.

\section{Local Extrema}

As one can learn from experiments, the adaptation evolution curves do not always converge to the same stationary state maximum or minimum that displays the presence of local extrema of the beam-quality metric as seen in Fig. 11. The existence of local maxima is perhaps one of the most difficult problems for adaptive wave-front control techniques based on optimization of the system performance metric. ${ }^{14-16}$

The following problems should be addressed to re-
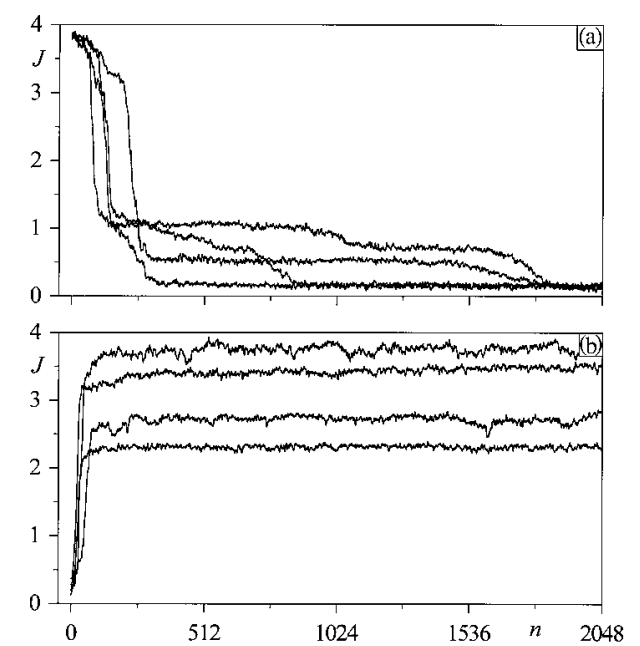

Fig. 11. Adaptation evolution curves for beam-quality metric (a) minimization and (b) maximization for a tip-tilt mirror array $\left(\mathrm{BU}_{\mathrm{tt}}\right)$ adaptive system. Curves correspond to four adaptation trials. veal the potential negative effect of the local extrema on adaptive-system performance: (1) Origin of local extrema and their dependence on type and resolution of the adaptive mirror.

(2) Frequency of occurrence (probability of occurrence) of local extrema and the difference (distance) between local and global metric values.

The origin of local maxima can be illustrated with the single-element sensitivity curves presented in Fig. 4. Assume that at some moment $t$ all elementsactuators of a $\mu$ mirror are aligned properly, except a single element [curve 2 in either Fig. 4(a) or 4(b)]. The state of this adaptive system corresponds to point A on the sensitivity curve in Fig. 4. Maximization of the beam-quality metric with a gradient ascent technique will result in the system's transitioning to the state that corresponds to the closest local maximum (point $\mathrm{A}^{\prime}$ in Fig. 4). For a tip-tilt mirror [Fig. 4(b)] the metric value (Strehl ratio) at the local maximum is significantly less than at the global maximum (perfectly adjusted system). For a piston-type mirror [Fig. 4(a)] the situation is different. The Strehl ratio at the local maximum has almost the same value as at the global maximum. For a perfect piston-type mirror, not all local maxima are distinguished. This situation corresponds to $2 \pi$-degenerate local maxima that have the same (optimal) Strehl ratio St values. Degeneracy of the local maxima is absent for tip-tilt and continuously deformable surface mirrors.

One can decrease the effects of local maxima by increasing the perturbation amplitudes $\left\{\delta u_{j}^{(n)}\right\}$ applied to the mirror electrodes, by increasing update coefficient $\gamma$, or both. In both cases the system has a higher probability of not getting trapped in the vicinity of the small-amplitude local maxima that compose the majority of the total local maxima. The adaptation process rather will converge to the global maximum or to a local maximum that corresponds to a metric value that is only slightly different from the global value. Increasing the perturbation amplitude and the update coefficient, however, may cause such negative effects as unwanted metric oscillation in the vicinity of a maximum.

The problem of local maxima can be analyzed by calculation of probability distribution $p(J)$ for beamquality metric $J$. The transition processes (from metric maximization to minimization and vice versa) may affect the accuracy of the calculated probability distributions. To avoid this effect we estimated probability distribution $p(J)$, using measured values of beam-quality metric $J(n)$ for the last 1000 iterations of metric maximization $p_{\max }(J)$ and minimization $p_{\min }(J)$ collected from all 500 adaptation trials: $p(J) \Delta J=N_{J} / N_{0}$, where $N_{J}$ is the number of cases that correspond to the beam-quality metric that belongs to the range $J \leq J<J+\Delta J$ and $N_{0}$ is the entire number of stored values of metric $J$. The minimal metric interval used for data collection corresponded to $\Delta J=J_{\max } / 400\left(J_{\max }=\max J\right)$. Probability curves for the beam-quality metric maximization and minimization in Fig. 12(a) display the presence of local extrema only for the adaptive system with the 

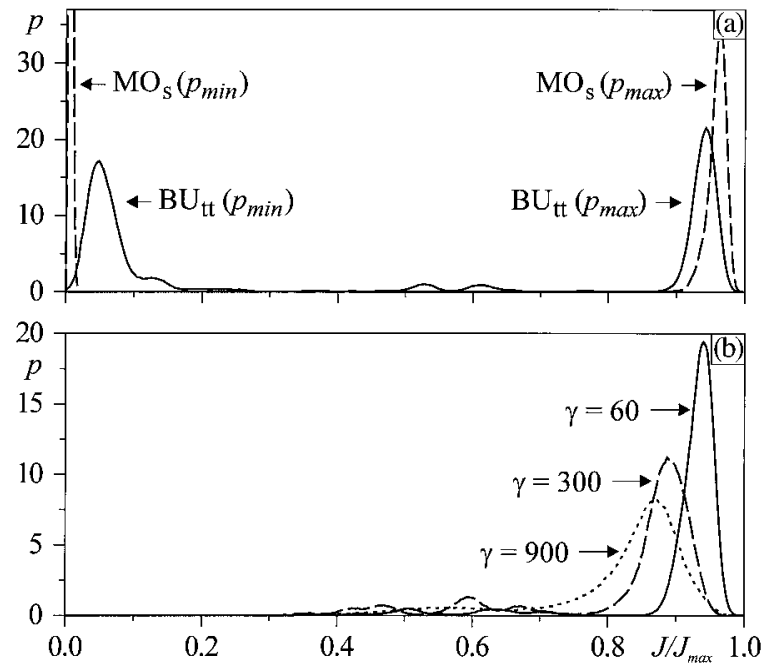

Fig. 12. Probability-density distributions of beam-quality metrics $p_{\max }$ and $p_{\min }$ obtained during 500 adaptation trials: (a) adaptive system with the $\mathrm{MO}_{s}$ and $\mathrm{BU}_{\mathrm{tt}} \mu$ mirrors and fixed update coefficient $\gamma=60$ and (b) probability distribution $p_{\max }$ for the adaptive system with the $\mathrm{BU}_{\mathrm{tt}} \mu$ mirror for three values of the update coefficient $\gamma$. Beam-quality metric $J$ is normalized by its maximum value $J_{\max }$.

tip-tilt type $\mathrm{BU}_{\mathrm{tt}}$ mirror. The probabilities for these local extrema are relatively small. As expected, the probability distributions for the adaptive system with the piston-type $\mathrm{MO}_{s}$ mirror are unimodal: All local extrema are $2 \pi$ degenerate and have approximately the same metric values. In the case of the tip-tilttype $\mathrm{BU}_{\mathrm{tt}}$ mirror, increasing the update coefficient value $\gamma$ eliminated local states in the adaptive system's dynamics but at the expense of widening the probability-density curves because of the increase in metric oscillations that occurs in the vicinity of the global maximum, as shown in Fig. 12(b).

\section{Control of Adaptation Rate: Change in Update Coefficient through Learning}

As we mentioned in Subsection 3.B, the system's adaptation rate depends on the value of update coefficient $\gamma$ that is externally supplied to the VLSI chips. Thus the AdOpt control system architecture permits on-the-fly (at each iteration) control of the update coefficient. For control of coefficient $\gamma$ the following information was available: the perturbed beamquality metric values $J_{+}^{(n)}$ and $J_{-}^{(n)}$ measured at each iteration and the calculated difference (metric perturbation) $\delta J^{(n)}=J_{+}^{(n)}-J_{-}^{(n)}$. In the experiments described below we also performed at each iteration an additional measurement of the unperturbed metric value $J^{(n)}$ and calculated two additional metric perturbations: $\quad \Delta J_{+}^{(n)}=J_{+}^{(n)}-J^{(n)}$ and $\Delta J_{-}^{(n)}=J_{-}^{(n)}-$ $J^{(n)}$. The measurement of $J^{(n)}$ lasted $\sim 25 \mu \mathrm{s}$. In the absence of the introduced time delay $\tau_{\text {del }}$ this additional measurement resulted in a nearly $15 \%$ increase in the time $\tau_{\text {it }}$ required for a single iteration. A portion of the obtained data was saved in computer memory, thus permitting the use of data from previous $l=1, \ldots, L$ iterations (control with $L$-step-long

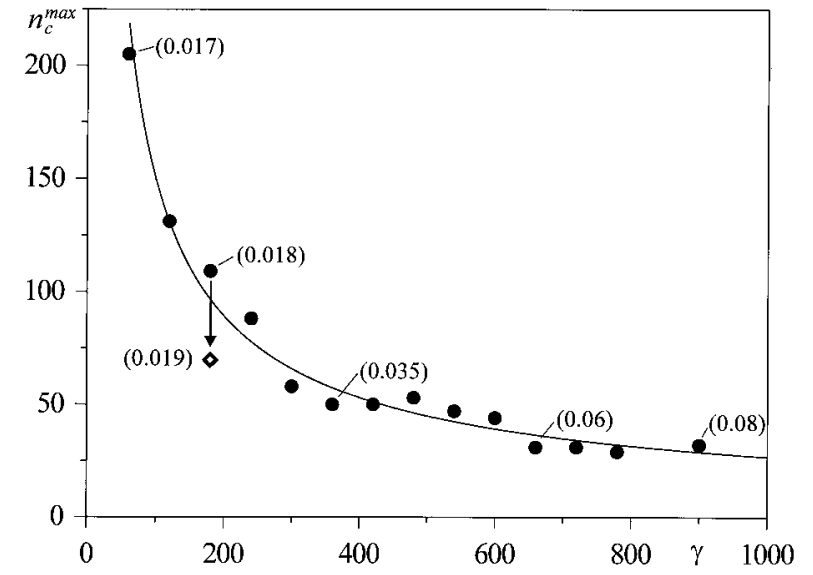

Fig. 13. Convergence rate $n_{c}{ }^{\text {max }}$ of the adaptation process relative to update coefficient $\gamma$ for maximization of the beam-quality metric in an adaptive system with the $\mathrm{BU}_{\mathrm{tt}}$ mirror. The metric fluctuation level is characterized by the normalized standard-deviation values $\bar{\sigma}_{J}$ shown in parentheses. The diamond represents results obtained in the system with a change in the adaptive update coefficient. The fluctuation level in the system without adaptation was near 0.01 .

memory). With a 400-MHz $\mathrm{PC}$ computer the additional time required for the calculations and data storage was less then $1.5 \mu \mathrm{s}$ and can be neglected. The question is how to use this available information to control the update coefficient.

First, consider the dependence of the adaptation process's convergence rate $n_{c}$ max (number of iterations required to reach $80 \%$ of the beam-quality metric's maximum value) on the update coefficient $\gamma$ as presented in Fig. 13. As expected, an increase in $\gamma$ resulted in acceleration of the convergence speed of the adaptation ( $n_{c}{ }^{\max }$ decrease). However, decreasing $n_{c}$ max increased the normalized standard deviation of beam-quality metric fluctuations, defined as $\bar{\sigma}_{J}=0.001 \sum_{n=3097}^{4096}\left\{\left\langle\left[(J(n)-\langle J(n)\rangle]^{2}\right\rangle^{1 / 2} /\langle J(n)\rangle\right\}\right.$. Standard deviation $\bar{\sigma}_{J}$ included averaging over the last 1000 iterations of each optimization trial. The values $\bar{\sigma}_{J}$ obtained in the experiment are shown in parentheses in Fig. 13. Increasing $\gamma$ is advantageous only during the transition phase of the adaptation process and is unfavorable when the adaptive system is near the extremum.

It follows that adaptive control should provide for an automatic increase in the update coefficient during the transition phase and for a decrease otherwise. The problem is to determine the current phase of the adaptation process (transition, near minimum, near maximum) and use this information for iterative change of the upgrade coefficient. We used the following three quantities (indicators) $H^{(n)}$ to identify phase in the adaptation process:

$$
\begin{aligned}
& H_{1}^{(n)}=\left|\operatorname{sign}\left[\Delta J_{+}^{(n)}\right]-\operatorname{sign}\left[\Delta J_{-}^{(n)}\right]\right|, \\
& H_{2}^{(n)}=\left|\delta J^{(n)}\right|, \\
& H_{3}^{(n)}=\sum_{l=1}^{L}\left|J^{(n)}-J^{(n-l)}\right| .
\end{aligned}
$$


It is easy to see that at the transition phase all $H^{(n)}$ indicators have higher values (on average) than the corresponding values for adaptive-system operation near an extremum. The length of memory $L$ in Eqs. (2) should be less than the typical convergence rate $n_{c}{ }^{\max }$ of the adaptation process. In the experiments we used $L=5$. To decrease the influence of noise we averaged the quantities in Eqs. (2) over a few iterations $M_{1}=5$ :

$$
\bar{H}_{i}^{(n)}=\frac{1}{M_{1}} \sum_{m=0}^{M_{1}} H_{i}^{(n-m)}, \quad i=1,2,3 .
$$

For a better understanding of the iterative algorithm used for update coefficient control, consider its continuous-time analog:

$$
\tau \frac{\mathrm{d} \gamma}{\mathrm{d} t}=\left(\gamma_{0}-\gamma\right)+\varepsilon_{0} \bar{H}(t),
$$

where $\bar{H}(t)=\bar{H}_{1}(t) \bar{H}_{2}(t) \bar{H}_{3}(t)$ and $\tau, \gamma_{0}$, and $\varepsilon_{0}$ are constants (algorithm parameters). In Eq. (4), continuous time $t$ is used instead of iteration number $n$. The introduced function $\bar{H}(t)$ is the product of all three indicators defined in Eq. (3). For adaptivesystem operation near an extremum (maximum or minimum) the function $\bar{H}_{1}$ vanishes because in the absence of noise the perturbations $\Delta J_{+}^{(n)}$ and $\Delta J_{-}^{(n)}$ have the same sign and their difference (the function on which $\bar{H}_{1}$ depends) is zero. Correspondingly, update coefficient $\gamma$ in Eq. (4) approaches the constant value $\gamma_{0}$. At the transition phase the indicator function $\bar{H}(t)$ is positive and increases with increase of the transition process slope. In the accordance with Eq. (4) this procedure creates an increase in update coefficient $\gamma$. The dynamic process of Eq. (4) represents a kind of learning rule that is reminiscent of continuous-type equations in neural network models for changes in weight coefficient through learning (called long-term memory traces). ${ }^{20}$

The following discrete analog of control algorithm (5) was used in the experiments to control the update coefficient during the adaptation process:

$$
\gamma^{(n+1)}=\gamma^{(n)}+\alpha\left[\gamma_{0}-\gamma^{(n)}\right]+\varepsilon \bar{H}_{1}^{(n)} \bar{H}_{2}^{(n)} \bar{H}_{3}^{(n)},
$$

where $\alpha$ and $\varepsilon$ are coefficients (in the experiments we used $\gamma_{0}=180, \alpha=0.9$, and $\varepsilon=1500$ ). Results of the adaptation with the adaptive-update coefficient change through learning are shown in Fig. 14. The averaged adaptation evolution curve $\langle J(t)\rangle$ for the system with the $\mathrm{BU}_{\mathrm{tt}}$ mirror is compared in Fig. 14(a) with the evolution curve that corresponds to constant update coefficient $\gamma=\gamma_{0}$. The dynamics of the update coefficient $\langle\gamma(t)\rangle$ averaged over all adaptation trials are shown in Fig. 14(b). During the transition stage, $\gamma$ increased $\sim 5.8$ times compared with the initial value $\gamma_{0}$. One can estimate the gain in the convergence speed of the adaptation process by comparing the characteristic adaptation times $\tau_{\text {ad }}$ and $\tau_{\text {ad }}^{(\gamma)}$ shown in Fig. 14(a) for the system with constant $\left(\tau_{\mathrm{ad}}\right)$ and adaptive $\gamma\left[\tau_{\mathrm{ad}}^{(\gamma)}\right]$ times. The measured ratio $\tau_{\mathrm{ad}} / \tau_{\mathrm{ad}}^{(\gamma)}$ indicates a 1.5 increase in the adaptation
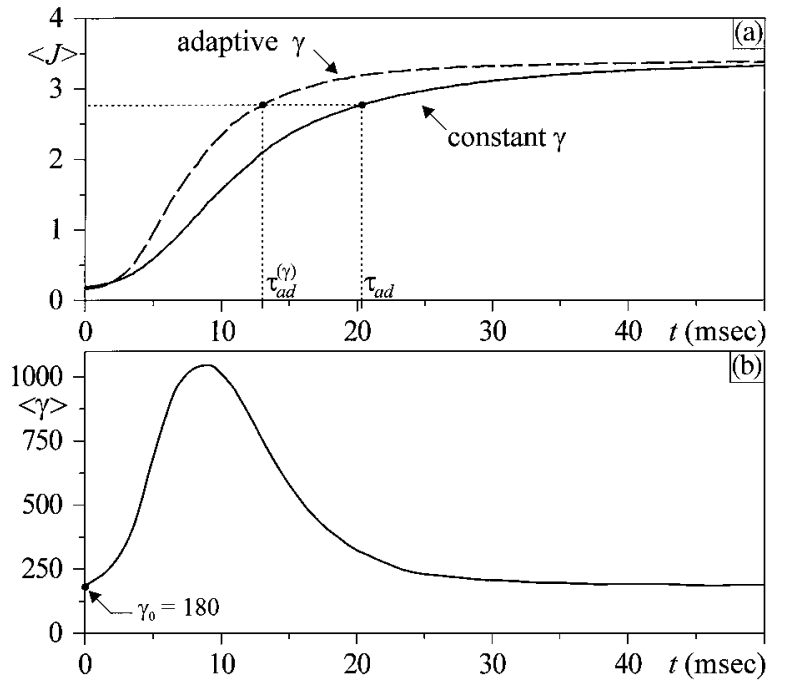

Fig. 14. Adaptive system with a change in the adaptive update parameter through learning rule (4): (a) beam-quality metric evolution curve averaged over 1000 trials and (b) corresponding evolution curve for update coefficient $\gamma$. The value $\gamma_{0}=180$ was used in the experiments, with fixed $\gamma$.

speed. The convergence rate that corresponds to the system with adaptive $\gamma$ is labeled with a diamond in Fig. 13. As the related number in parentheses in Fig. 13 indicates, improvement in the convergence rate was obtained without an increase of the metric scintillation amplitude.

\section{Conclusion}

We have demonstrated microscale adaptive-optics systems formed by combining recently developed micromachined mirror arrays with a VLSI stochastic parallel gradient descent controller. A record adaptation rate of 6000 iterations/s was achieved with the Boston University mirror array. This system required the use of external amplifiers. We also demonstrated a microscale adaptive system by directly interfacing the low-voltage $\mu$-mirror array from MEMS Optical, Inc., with the VLSI controller. The adaptive systems considered here with both tip-tiltand piston-type micromachined mirrors arrays have demonstrated efficient compensation for self-induced wave-front phase distortions.

The MEMS-VLSI-based wave-front control technique presented here has several attractive features, as follows:

(1) Adaptive optics based on the model-free optimization wave-front control technique do not require either a wave-front sensor or computationally expensive wave-front reconstruction hardware. Thus the development of compact, low-power, and inexpensive adaptive systems composed solely of microscale components is facilitated. In this respect the further development of lower-voltage $\mu$-mirror arrays directly driven by a VLSI controller as well as the integration of high-voltage amplifiers onto a $\mu$-mirror 
chip are important directions for further research for microscale adaptive optics.

(2) Micromachined mirror arrays with multikilohertz operational bandwidths are capable of performing tens of thousands of iterations per second. This is exactly what iteration-hungry, modelfree optimization-based adaptive optics needs for compensation of quickly changing atmosphericturbulence-induced wave-front aberrations. As we have demonstrated, the convergence rate of the adaptation process can also be increased by incorporation of an additional long-term memory learning feedback loop.

(3) Both the micromachined mirror arrays and the VLSI stochastic gradient descent controller are scalable microscale components that facilitate the development of high-resolution adaptive systems with thousands of wave-front phase-control channels. A potential obstacle to this development is the so-called wiring problem. The ability to control mirror elements in parallel is the major advantage of the stochastic gradient optimization technique and should be preserved during a transition to high-resolution adaptive systems. Possible solutions of the wiring problem are on-chip integration of the VLSI controller with $\mu$-mirror arrays and flip-chip bonding of $\mu$-mirror and VLSI controller chips.

The authors thank Victor Bright, who generously offered the $\mu$-mirror array designed at the University of Colorado for examination in our experiments, Gary Carhart for assisting with the computer code, and Jennifer Ricklin for technical and editorial comments. This research was performed at the U.S. Army Research Laboratory's Intelligent Optics Lab. Research was supported in part through the following U.S. Army and U.S. Air Force programs: Cooperative Agreement DAAD17-99-2-0070 between the U.S. Army Research Laboratory and the Boston University Photonic Center; grant DAAG55-97-1-0114 through the U.S. Army Research Office under the ODDR\&E MURI97 program to the Center for Dynamics and Control of Smart Structures (through Harvard University); U.S. Air Force Office of Scientific Research contract F49620-99-1-0342 with New Mexico State University; and U.S. Army Research Office Small Business Innovative Research grant A98-021 to MEMS Optical, Inc. T. Weyrauch currently holds a National Research Council Research Associateship Award at the U.S. Army Research Laboratory.

\section{References}

1. N. Maluf, An Introduction to Microelectromechanical Systems Engineering (Artech House, Norwood, Mass., 2000).

2. M. C. Wu, "Micromachining for optical and optoelectronic systems," Proc. IEEE 85, 1833-1856 (1997).

3. R. L. Clark, J. R. Karpinisky, J. A. Hammer, R. B. Anderson, R. L. Lindsey, D. M. Brown, and P. H. Merritt, "Micro-optoelectro-mechanical (MOEM) adaptive optic system," in Miniaturized Systems with Micro-Optics and Micromechanics II, M. E. Motamedi, L. J. Hornbeck, and K. S. Pister, eds., Proc. SPIE 3008, 12-24 (1997).

4. T. G. Bifano, R. Krishnamoorthy Mali, J. K. Dorton, J. Per- reault, N. Vandelli, M. N. Horenstein, and D. A. Castañon, "Continuous-membrane surface-micromachined silicon deformable mirror," Opt. Eng. 36, 1354-1360 (1997).

5. T. G. Bifano, J. Perrault, R. Krishnamoorthy Mali, and M. N. Horenstein, "Microelectromechanical deformable mirrors," IEEE J. Sel. Top. Quantum Electron. 5, 83-89 (1999).

6. J. A. Hammer, J. Karpinsky, R. L. Clark, and R. Lindsey, "Micro mirrors in adaptive optics systems," in Proceedings of the World Automation Congress (WAC '98), M. Jamshidi and C. W. de Silva, eds., Vol. 6 of TSI Press Series (TSI, Albuquerque, N.M., 1998), pp. 575-581.

7. M. K. Lee, W. D. Cowan, B. M. Welsh, V. M. Bright, and M. C. Roggemann, "Aberration-correction results from a segmented microelectromechanical deformable mirror and refractive lenslet array," Opt. Lett. 23, 645-647 (1998).

8. G. Vdovin, S. Middelhoek, and P. M. Sarro, "Technology and applications of micromachined silicon adaptive mirrors," Opt. Eng. 36, 1382-1390 (1997).

9. J. H. Comtois, V. M. Bright, S. C. Gustafson, and M. A. Michalicek, "Implementation of hexagonal micromirror arrays as phase-mostly spatial light modulator," in Microelectronic Structures and Microelectromechanical Devices for Optical Processing and Multimedia Applications, W. Bailey, M. E. Motamedi, and F.-C. Luo, eds., Proc. SPIE 2641, 76-87 (1995).

10. J. Mansell and R. L. Byer, "Micromachined silicon deformable mirror," in Adaptive Optical System Technologies, D. Bonaccini and R. K. Tyson, eds., Proc. SPIE 3353, 896-901 (1998).

11. J. C. Spall, "A stochastic approximation technique for generating maximum likelihood parameter estimates," in Proceedings of the American Control Conference (Institute of Electrical and Electronics Engineers, Piscataway, N.J., 1987), pp. 1161-1167.

12. G. Cauwenberghs, "A fast stochastic error-descent algorithm for supervised learning and optimization," in Advances in Neural Information Processing Systems, S. J. Hanson, J. D. Cowan, and C. L. Giles, eds. (Morgan Kaufman, San Mateo, Calif., 1993), Vol. 5, pp. 244-251.

13. M. A. Vorontsov, G. W. Carhart, and J. C. Ricklin, "Adaptive phase-distortion correction based on parallel gradient-descent optimization," Opt. Lett. 22, 907-909 (1997).

14. M. A. Vorontsov and V. P. Sivokon, "Stochastic parallel gradient descent technique for high-resolution wave-front phase distortion correction," J. Opt. Soc. Am. A 15, 2745-2758 (1998).

15. R. T. Edwards, M. Cohen, G. Cauwenberghs, M. A. Vorontsov, and G. W. Carhart, "Analog VLSI parallel stochastic optimization for adaptive optics," in Learning on Silicon, G. Cauwenberghs and M. A. Bazoumi, eds. (Kluwer Academic, Boston, Mass., 1999), pp. 359-382.

16. M. A. Vorontsov, G. W. Carhart, M. Cohen, and G. Cauwenberghs, "Adaptive optics based on analog parallel stochastic optimization: analysis and experimental demonstration," J. Opt. Soc. Am. A 17, 1440-1453 (2000).

17. A. Tuantranont, V. M. Bright, W. Zhang, and Y. C. Lee, "Flip chip integration of lenslet arrays on segmented deformable micromirrors," in Design, Test, and Microfabrication of MEMS and MOEMS, B. Courtois, S. B. Crary, W. Ehrfeld, H. Fujita, J.-M. Karam, and K. W. Markus, eds., Proc. SPIE 3680, 668-678 (1999).

18. M. C. Roggeman, V. M. Bright, B. M. Welsh, S. R. Hick, P. C. Roberts, W. D. Cowan, and J. H. Comtois, "Use of microelectro-mechanical deformable mirrors to control aberrations in optical systems: theoretical and experimental results," Opt. Eng. 36, 1326-1338 (1997).

19. L. Zhu, P.-C. Sun, D.-U. Bartsch, W. R. Freeman, and Y. Fainman, "Adaptive control of a micromachined continuousmembrane deformable mirror for aberration compensation," Appl. Opt. 38, 168-176 (1999).

20. S. Grossberg, "Nonlinear neural networks: principles, mechanisms, and architectures," Neural Networks 1, 17-61 (1988). 